\title{
Crescimento de mudas de Mimosa scabrella Benth em função de diferentes tamanhos de recipientes e doses de fertilizante ${ }^{1}$
}

\author{
Ângela Maria Stüpp ${ }^{2}$; Marcio Carlos Navroski ${ }^{3}$; Dionéia Felippe ${ }^{4}$; Daniella Del Castanhel Kniess ${ }^{5}$; \\ Julio Cesar Amancio ${ }^{6}$; Matheos Abner Silva ${ }^{7}$; Mariane de Oliveira Pereira ${ }^{8}$
}

\begin{abstract}
Resumo: O presente trabalho objetivou avaliar diferentes tamanhos de tubetes e doses de fertilizante de liberação controlada para a produção de mudas de bracatinga. Foram utilizados tubetes de $110 \mathrm{~cm}^{3} \mathrm{e} 180 \mathrm{~cm}^{3}$, preenchidos com substrato comercial Tecnomax ${ }^{\circledR} \mathrm{e}$ adicionadas as doses $0,3,6 \mathrm{e} 9 \mathrm{~g} \mathrm{~L}^{-1}$ de fertilizante Osmocote ${ }^{\circledR}$. O experimento foi realizado em delineamento inteiramente casualizado (DIC), em esquema bifatorial $2 \times 4$. Foram utilizadas quatro repetições, cada uma composta por 16 plantas. As avaliações foram feitas 96 dias após a semeadura. A análise morfológica foi realizada por meio de medições de altura da parte aérea $(\mathrm{cm})$, diâmetro de colo $(\mathrm{mm})$, bem como a relação entre altura da parte aérea e diâmetro de colo (H/D). A dosagem de fertilizante que se mostrou mais eficiente foi de $6 \mathrm{~g} \mathrm{~L}^{-1}$, e o tubete que promoveu resultado mais satisfatório foi de $180 \mathrm{~cm}^{3}$. As doses de adubo de liberação lenta influenciaram o crescimento inicial das mudas de bracatinga, demostrando potencial de uso na adubação da espécie.
\end{abstract}

Palavras - chave: Bracatinga; Produção de mudas; Nutrição de mudas; Osmocote ${ }^{\circledR}$.

\section{Mimosa scabrella Benth seedling growth in different recipients size and fertilizers doses function}

\begin{abstract}
This study evaluated different sized tubes and controlled release fertilizer doses to produce Mimosa scabrella seedlings. Tubes of $110 \mathrm{~cm}^{3}$ and $180 \mathrm{~cm}^{3}$ were used, filled with commercial substrate Tecnomax ${ }^{\circledR}$ and added $0,3,6$ and $9 \mathrm{~g}$ $\mathrm{L}^{-1}$ Osmocote ${ }^{\circledR}$. The experiment was conducted in a completely randomized design, in a factorial $2 \times 4$. We used four replicates, each consisting of 16 plants. Evaluations were made 96 days after sowing. Morphologic analysis was performed by means of shoot height measurements $(\mathrm{cm})$, stem diameter $(\mathrm{mm})$ and the ratio of shoot height and diameter $(\mathrm{H} / \mathrm{D})$. The dosage of fertilizer that is more efficient was $6 \mathrm{~g} \mathrm{~L}^{-1}$, and the cartridge that had the most satisfactory result was $180 \mathrm{~cm}^{3}$. The slow-release fertilizer levels influenced the early growth of seedlings Mimosa scabrella, showing potential use in the fertilization of the specie
\end{abstract}

Keywords: Mimosa scabrella Benth; Seedling production; Nutrition seedlings; Osmocote.

\footnotetext{
${ }^{1}$ Recebido em 29.06.2015 e aceito para publicação como artigo científico em 06.10.2015.

${ }^{2}$ Engenheira Florestal, Mestranda do Programa de Pós-graduação em Engenharia Florestal do Centro de Ciências Agroveterinárias da Universidade do Estado de Santa Catarina (CAV-UDESC). E-mail: <angela.stupp@ hotmail.com>.

${ }^{3}$ Engenheiro Florestal Dr., Professor do Centro de Ciências Agroveterinárias (CAV-UDESC).

${ }^{4}$ Engenheira Florestal, Mestranda do Programa de Pós-graduação em Engenharia Florestal do Centro de Ciências Agroveterinárias da Universidade do Estado de Santa Catarina (CAV-UDESC).

${ }^{5}$ Engenheira Florestal, Mestranda do Programa de Pós-graduação em Engenharia Florestal do Centro de Ciências Agroveterinárias da Universidade do Estado de Santa Catarina (CAV-UDESC).

${ }^{6}$ Engenheiro Florestal pela Universidade do Estado de Santa Catarina, (CAV/UDESC).

${ }^{7}$ Engenheiro Florestal pela Universidade do Estado de Santa Catarina, (CAV/UDESC).

${ }^{8}$ Engenheira Florestal, Doutoranda do Programa de Pós-graduação em Engenharia Florestal da Universidade Federal do Paraná.
} 


\section{Introdução}

Mimosa scabrella Benth. conhecida popularmente como bracatinga é uma espécie da família Fabaceae, pertencente à subfamília Mimosoideae. Esta espécie ocorre desde Minas Gerais até a borda da Serra Geral, no Rio Grande do Sul, caracterizando o planalto sul brasileiro e a Floresta Ombrófila Mista, ocorrendo também na Floresta Atlântica e Floresta Estacional do Alto Uruguai (MARCHIORI, 2007).

É uma árvore perenifólia pioneira, característica das regiões mais frias do sul do Brasil, onde forma com frequência povoamentos puros, de rápido crescimento quando comparada com outras espécies florestais nativas (LORENZI, 1998).

A espécie apresenta interesse do ponto de vista econômico, para lenha e confecção de móveis e, ecológico, na recuperação de áreas degradadas e em consórcios agroflorestais (ROSA, 2009). Seu plantio é recomendado também para proteger e recuperar solos degradados, erodidos ou com baixa fertilidade, em curto prazo, por cobri-los rapidamente e fixar nitrogênio (CARNEIRO et al., 1982).

Nas áreas plantadas por sementes ou por mudas, o crescimento responde à profundidade efetiva e à riqueza química dos solos, particularmente à adição de fósforo. A espécie também tolera terrenos pedregosos e terraplanados (CARPANEZZI, 1994).

A formação de mudas é um dos pontos determinantes do processo de produção, o qual pode possibilitar a obtenção, em viveiro, de plantas com melhor desempenho para suportar as condições adversas de campo. Expressivos aumentos no crescimento e qualidade de mudas podem ser alcançados através da adubação mineral, (BARBOSA et al., 2003).

O cultivo em substrato distingue-se do cultivo em solo principalmente pela área limitada para o desenvolvimento das raízes. A limitação do volume exige que o substrato seja capaz de manter água facilmente disponível à planta sem comprometer a concentração de oxigênio no meio (FERMINO, 2002).

O substrato apresenta papel fundamental, pois deve fornecer às mudas todas as condições químicas, físicas e biológicas para um crescimento saudável, oferecendo assim condições de transformar seu potencial genético em produtividade (KÄMPF e FERMINO, 2000). Por isso, aliado a um bom substrato, deve ser utilizado um fertilizante com alta qualidade, em doses adequadas e preferencialmente com mecanismos de liberação controlada de nutrientes, evitando perdas por lixiviação e volatilização. Devem-se conduzir esforços para a utilização de substratos que permitam o maior desenvolvimento e qualidade das mudas (SIMÕES et al., 2012).

Os fertilizantes de liberação controlada são alternativas interessantes, pois permitem que os nutrientes sejam disponibilizados de maneira contínua à planta, minimizando os riscos de deficiências (MENDONÇA et al., 2004). Outras vantagens relacionam-se à redução de custos operacionais, já que a aplicação é realizada uma única vez. Além disto, também há diminuição da salinidade do substrato, a qual poderia prejudicar o pleno desenvolvimento da muda (SHARMA, 1979).

Nas últimas décadas presenciou-se a mudança do método de produção de mudas, passando de embalagens tradicionais como os sacos plásticos para o sistema de tubetes, o que dentre outras vantagens, trouxe a possibilidade de mecanização das operações de produção. Devido a menor quantidade de substrato, principalmente de base orgânica, utilizado neste tipo de recipiente, há a obrigatoriedade de adubações, que devem ser frequentes. Além disso, devido à alta lixiviação de nutrientes, há necessidade de adubações em cobertura (GONÇALVES et al., 2000).

Portanto, o tamanho do recipiente para a produção de mudas em viveiros florestais tem influência direta em seu custo final, pois deste resulta a quantidade de substrato a ser utilizado, o espaço que ocupado no viveiro, a mão de obra utilizada no transporte, remoções para aclimatação e retirada para entrega ao produtor, além da influência na quantidade de insumos e/ou fertilizantes e água que irá demandar (QUEIROZ e MELÉM JÚNIOR, 2001). 
Neste contexto, este trabalho teve como objetivo avaliar doses crescentes de fertilizante de liberação controlada em tubetes de diferentes tamanhos no crescimento inicial de mudas de Mimosa scabrella.

\section{Material e métodos}

O experimento foi conduzido no Viveiro Florestal do Departamento de Engenharia Florestal do Centro de Ciências Agroveterinárias - CAV, da Universidade do Estado de Santa Catarina-UDESC, no período de março a junho de 2013.

De acordo com a classificação de Köppen o clima do município de Lages/SC é mesotérmico úmido com verão ameno ( $\mathrm{Cfb}$ - temperado). A temperatura média anual é $15^{\circ} \mathrm{C}$ e a precipitação média anual é de $1300 \mathrm{~mm}$ a $1500 \mathrm{~mm}$.

As sementes de Mimosa scabrella Benth foram coletadas de 10 árvores nativas localizadas próximo à fazenda do $\mathrm{CAV}$ - Centro de Ciências Agroveterinárias - UDESC, na localidade de Macacos, município de Lages, SC. Após, as sementes foram extraídas, beneficiadas de forma manual em peneiras de $2 \mathrm{~mm}$ de malha e acondicionadas em sacos de plástico, sendo após armazenadas durante seis meses em temperatura de 7 a $10^{\circ} \mathrm{C}$, até o início do experimento.

No planejamento dos tratamentos, utilizou-se o delineamento inteiramente casualizado (DIC), em esquema fatorial $2 \times 4$, em que os níveis do fator "A" referiram-se ao tamanho de tubete (110 $\mathrm{cm}^{3}$ e $180 \mathrm{~cm}^{3}$ ), e os níveis do fator "B", às doses do fertilizante de liberação controlada - FLC $\left(0 ; 3 ; 6 ; 9 \mathrm{~g} \mathrm{~L}^{-1}\right.$ de fertilizante). Foram utilizadas quatro repetições, cada uma composta por 16 plantas. Os tubetes de $110 \mathrm{~cm}^{3}$ possuíam $140 \mathrm{~mm}$ de altura, $40 \mathrm{~mm}$ de diâmetro e 6 estrias internas, e os de $180 \mathrm{~cm}^{3}$ possuíam $130 \mathrm{~mm}$ de altura, 54 $\mathrm{mm}$ de diâmetro e 8 estrias internas. O fertilizante de liberação controlada (FLC) foi o Osmocote $^{\circledR}$ na formulação 19-6-10 (NPK), caracterizado pela liberação lenta de 4 a 5 meses.

Para a superação da dormência, antes da semeadura as sementes foram submetidas à imersão em água quente, com aproximadamente $80^{\circ} \mathrm{C}$ por 18 horas. Os tubetes foram colocados em bandejas e organizados de acordo com seus tamanhos. Estes foram preenchidos com substrato comercial Tecnomax ${ }^{\circledR}$, composto por turfa, vermiculita expandida, casca de pinus e carvão vegetal, apresentando as seguintes características: $\mathrm{pH}=6,0( \pm 0,5)$; condutividade elétrica $=0,7( \pm 0,3) \mathrm{mS} \mathrm{cm}^{-1}$; densidade $=500$ $\mathrm{kg} \mathrm{m}^{-3}$; capacidade de retenção de água - CRA $(\mathrm{p} / \mathrm{p})=150 \%$ e umidade máxima $(\mathrm{p} / \mathrm{p})=50 \%$.

Juntamente com o substrato foram adicionadas as doses do fertilizante de liberação lenta (Osmocote $\AA$ ) de acordo com cada tratamento. A semeadura foi efetuada colocando-se duas a três sementes em cada tubete. Quando as plântulas atingiram aproximadamente 3 a $4 \mathrm{~cm}$ de altura, realizou-se o raleio, permanecendo a mais vigorosa e centralizada no recipiente. Para a cobertura das sementes foi utilizada uma camada fina de substrato contendo ou não fertilizante, assim especificado nos respectivos tratamentos, visando cobertura homogênea. Durante a condução do experimento, o regime hídrico na casa de vegetação foi sistematizado com duas irrigações diárias, com bicos microaspersores. Após a semeadura, as bandejas (dimensões $62 \mathrm{x}$ $42 \times 16 \mathrm{~cm}$ ) foram colocadas em casa de vegetação, sendo ainda, realizadas duas adubações de cobertura utilizando $6 \mathrm{~g} \mathrm{~L}^{-1}$ de ureia $(45 \% \mathrm{~N})$.

A análise morfológica foi realizada 96 dias após a semeadura, através de medições de altura da parte aérea $(\mathrm{cm})$, diâmetro de colo $(\mathrm{mm})$, bem como a relação entre altura da parte aérea e diâmetro de colo (H/D). Para a obtenção da altura da parte aérea foi utilizada uma régua milimetrada, medindo-se da base do colo até a gema apical que deu origem à última folha. $\mathrm{O}$ diâmetro do colo foi obtido por meio de um paquímetro digital de precisão. A avaliação foi realizada em todas as plantas do experimento.

As variâncias dos tratamentos foram testadas quanto à homogeneidade pelo teste de Bartlett. Quando houve homogeneidade das variâncias os dados foram submetidos à análise de variância e quando houve diferença significativa pelo teste 
de $\mathrm{F}$, houve aplicação de teste $\mathrm{T}$ e/ou regressão polinomial ao nível de $5 \%$ de probabilidade. A dose de máxima eficiência técnica (DMET) do FLC, referente a cada característica avaliada foi determinada a partir do cálculo das derivadas parciais das equações ajustadas pela análise de regressão. A análise dos dados foi realizada no pacote estatístico Sisvar (FERREIRA, 2011).

\section{Resultados e Discussão}

O uso de tubetes com diferentes capacidades volumétricas promoveram crescimentos diferentes em relação a dosagem do fertilizante. A altura das mudas variou em função das doses do fertilizante e do tamanho do tubete utilizado, apresentando comportamento quadrático (Figura 1). Resposta semelhante a este, foi descrito por Brondani et al. (2008), onde a altura das mudas de Anadenanthera colubrina (Veloso) Brenan, popularmente conhecido como angico-branco, variou aos 95 dias em função das doses de fertilizante de liberação controlada, também apresentando este comportamento.

Para as mudas cultivadas em tubetes de 180 $\mathrm{cm}^{3}$ observou-se que aquelas que receberam a dosagem de $6 \mathrm{~g} \mathrm{~L}^{-1}$ apresentaram maior crescimento e um melhor desempenho, correspondendo a uma altura de 23,22 cm, e um acréscimo superior a quatro vezes a altura do tratamento testemunha (substrato sem adição de Osmocote $\left.{ }^{\circledR}\right)$. A dose de máxima eficiência técnica (MET) calculada para a curva desta variável foi estimada em $6,25 \mathrm{~g} \mathrm{~L}^{-1}$.

Observou-se que o efeito do fertilizante foi positivo até uma determinada dose e, partindo desta, passou a ser negativo, ou seja, o excesso de Osmocote ${ }^{\circledR}$ passou a prejudicar o crescimento das mudas. Já para o tubete de $110 \mathrm{~cm}^{3}$, as dosagens de 3 e $9 \mathrm{~g} \mathrm{~L}^{-1}$ apresentaram resultados semelhantes, onde $9 \mathrm{~g} \mathrm{~L}^{-1}$ apresentou maior crescimento, correspondendo a uma altura de 21,6 cm, ou seja, 4 vezes superior à testemunha. Para esse volume de substrato a DMET foi estimada em 7,70 $\mathrm{g} \mathrm{L}^{-1}$.

Para ambos os tamanhos de tubete a diferença com o uso do FLC foi grande em relação ao tratamento sem adição do Osmocote $^{\circledR}$. Conforme José et al. (2005) o volume do tubete tem efeito significativo sobre as variáveis morfológicas e desta forma, é um fator limitante ao crescimento da muda. As dimensões dos recipientes e os consequentes volumes também influenciam a disponibilidade de nutrientes e água, devendo ser ressaltado que maior volume promove maior crescimento e melhora a arquitetura do sistema radicular. Em contrapartida, quanto maior os recipientes maiores os gastos com substrato, aumento da área do viveiro, e aumento dos custos de transporte das mudas (CARNEIRO, 1995).

Resposta similar no incremento em altura com aplicação de Osmocote ${ }^{\circledR}$ na produção de mudas de Guazuma ulmifolia, Peltophorum dubium, Eucalyptus grandis, Calycophyllum spruceanum e Pinus caribaea var. caribaea foi observado no trabalho de Moraes Neto et al. (2003), porém com amplitudes diferentes e em outras condições de trabalho. Para essas espécies, as doses compreendidas entre 4,3 e 6,4 $\mathrm{g} \mathrm{L} \mathrm{L}^{-1}$ de Osmocote ${ }^{\circledR}$ apresentaram os maiores valores em altura, aos 125 dias de avaliação.

Quanto ao crescimento em diâmetro de colo das mudas nos diferentes tamanhos de tubete em relação à dosagem do fertilizante de liberação controlada (Figura 2), observou-se que ocorreu interação entre os tratamentos, sendo que as dosagens de 3 e 9 g $\mathrm{L}^{-1}$ apresentaram comportamentos superiores em tubete de 110 $\mathrm{cm}^{3}$. O tratamento sem fertilizante não apresentou mudas com diâmetro de colo suficiente para atender ao critério.

A MET calculada para a curva desta variável foi de $6,54 \mathrm{~g} \mathrm{~L}^{-1}$. Todavia, as mudas plantadas nos tubetes de $180 \mathrm{~cm}^{3}$, tiveram melhor desempenho em diâmetro de colo, onde o melhor incremento nesta variável ocorreu no tratamento com a dosagem de $6 \mathrm{~g} \mathrm{~L}^{-1}$. Apenas o tratamento sem a dose de fertilizante não apresentou mudas com o diâmetro mínimo estabelecido pelo critério. A dose de máxima eficiência técnica para esta curva foi estimada em $6,80 \mathrm{~g} \mathrm{~L}^{-1}$.

Estudos realizados por Sturion (2000) com Mimosa scabrella comprovaram que as mudas cultivadas em recipientes com maior volume 
apresentaram maior diâmetro de colo, quando

recipientes de volume menor.

comparadas com aquelas cultivadas em

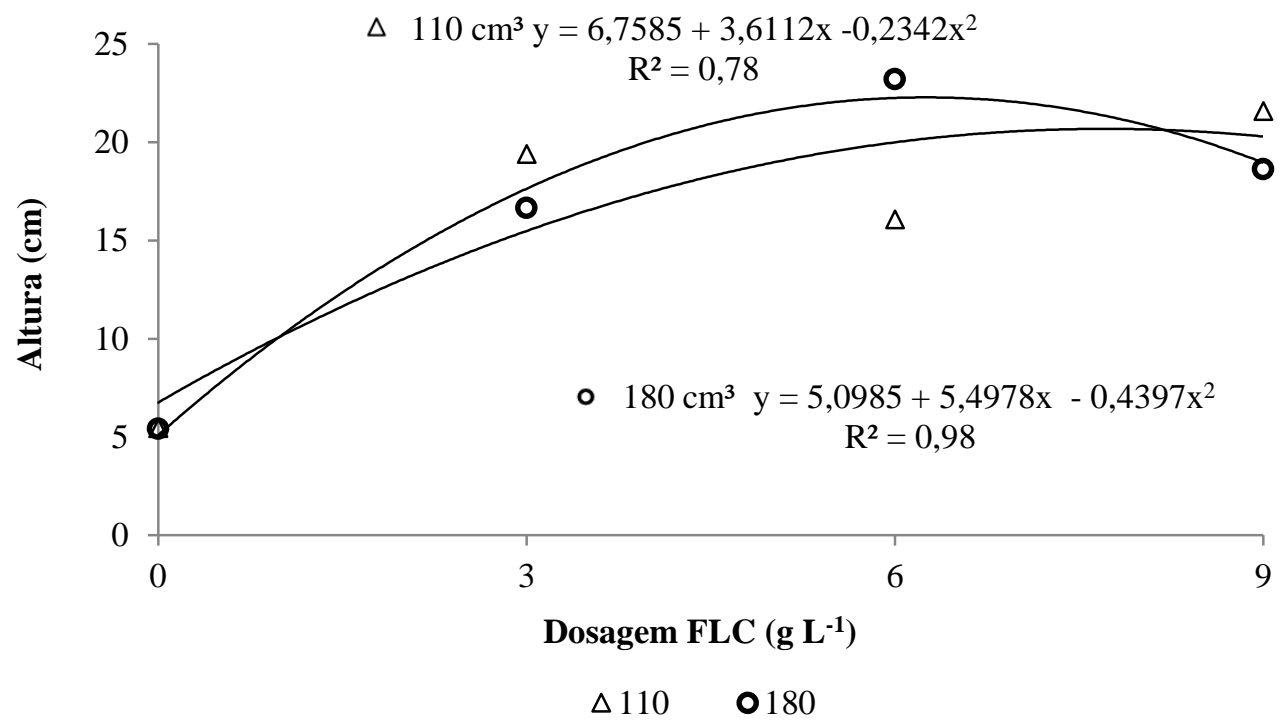

Figura 1: Altura (cm) de mudas de Mimosa scabrella, em relação à dosagem do fertilizante de liberação controlada (FLC) e volume dos tubetes.

Figure 1: Height $(\mathrm{cm})$ seedlings of Mimosa scabrella in relation to the dosage of the controlled release fertilizer (FLC) and the volume of the tubes.

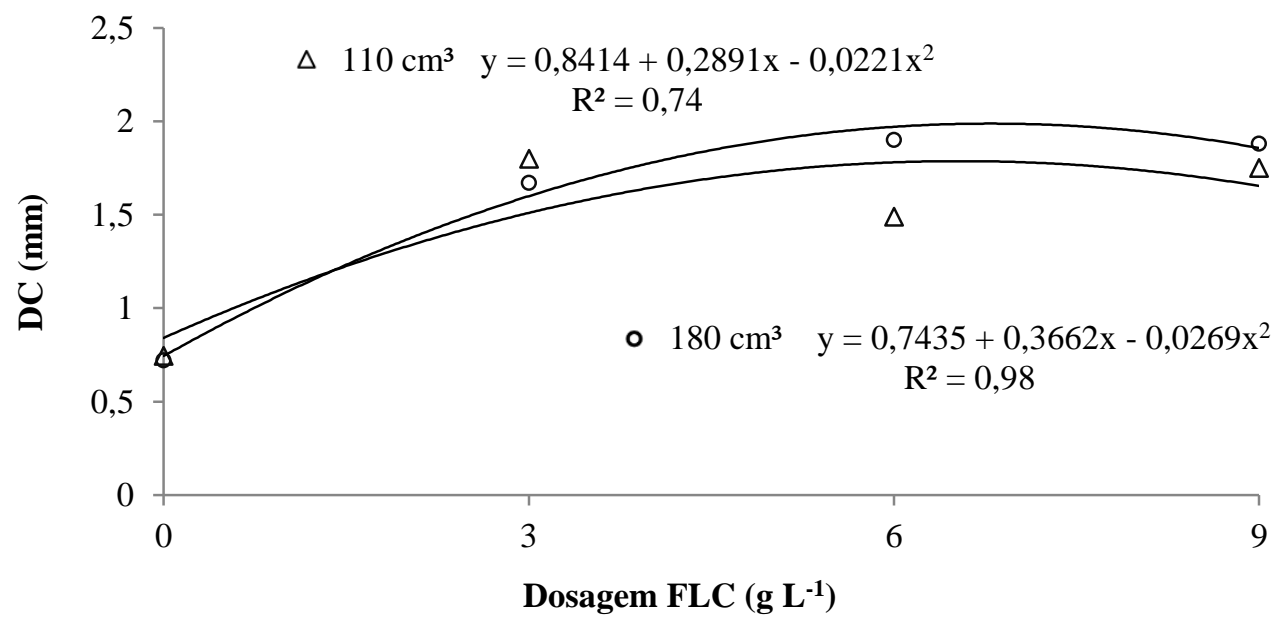

$\Delta 110 \quad \circ 180$

Figura 2: Diâmetro do colo $(\mathrm{mm})$ de mudas de Mimosa scabrella, em relação à dosagem do fertilizante de liberação controlada (FLC) e volume dos tubetes.

Figure 2: Stemdiameter $(\mathrm{mm})$ seedlings of Mimosa scabrella in relation to the dosage of the controlled release fertilizer (FLC) and volume of tubes.

Plantas com maior diâmetro proporcionam melhores condições de sobrevivência a campo, devido a maior competência de formação e crescimento de novas raízes (REIS et al., 1991). Geralmente, existe uma elevada correlação entre o aumento no diâmetro de colo e a sobrevivência 
de mudas depois do plantio (CARNEIRO, 1983).

Conforme relatam Jesus et al. (1987), as dimensões do recipiente e o tipo de substrato influenciam a qualidade das mudas, e isso se deve, possivelmente, ao fato de esses elementos estarem diretamente relacionados com a disponibilidade de espaço físico e o aporte de nutrientes, condições essenciais para o cultivo de plantas ex situ.

No comportamento da relação altura/diâmetro do colo das mudas nos diferentes tamanhos de tubete para as variadas dosagens do fertilizante de liberação lenta, constatou-se que houve interação entre os tratamentos, sendo que as a ausência do FLC proporcionou os menores valores (Figura 3), elevando a relação com a adição do fertilizante, contudo com tendência de estabilização da curva com o tubete $110 \mathrm{~cm}^{3} \mathrm{e}$ de diminuição da relação a partir do uso de $6 \mathrm{~g} \mathrm{~L}^{-1}$ de FLC com o uso dos tubetes de $180 \mathrm{~cm}^{3}$.

Cada espécie tem sua própria faixa de oscilação para relação H/DC. Carneiro (1995) recomenda que esses valores devem situar-se entre 5,4 e 8,1 para a espécie Pinus taeda, sendo, assim, mudas com desenvolvimento mais equilibrado entre as duas partes e com maior qualidade e capacidade de sobrevivência em campo. O autor comenta ainda que as mudas devem apresentar um diâmetro mínimo em relação à altura da parte aérea, e quanto menor for essa relação, melhor será a qualidade das mudas. Entretanto é normal a obtenção de valores superiores na relação H/DC, sem a perda de qualidade de mudas (KRATZ e WENDLING, 2013).

A relação altura/diâmetro do colo compõe um dos parâmetros usados para se avaliar a qualidade de mudas de espécies florestais, pois, além de refletir o acúmulo de reservas, assegura maior resistência e melhor fixação no solo. Geralmente, mudas com baixo diâmetro do colo apresentam dificuldades após o plantio (STURION, 2000). Essas dificuldades geralmente são representadas pela quebra na região do colo (gerando brotações laterais), crescimento de forma tortuosa ou ainda, mortalidade da planta. Essa variável é reconhecida como uma das mais importantes, se não a melhor indicadora do padrão de qualidade de mudas (MOREIRA e MOREIRA, 1996). Sendo assim, mudas com diâmetro do colo pequeno e alturas elevadas são consideradas de qualidade inferior às menores e com maior diâmetro do colo.

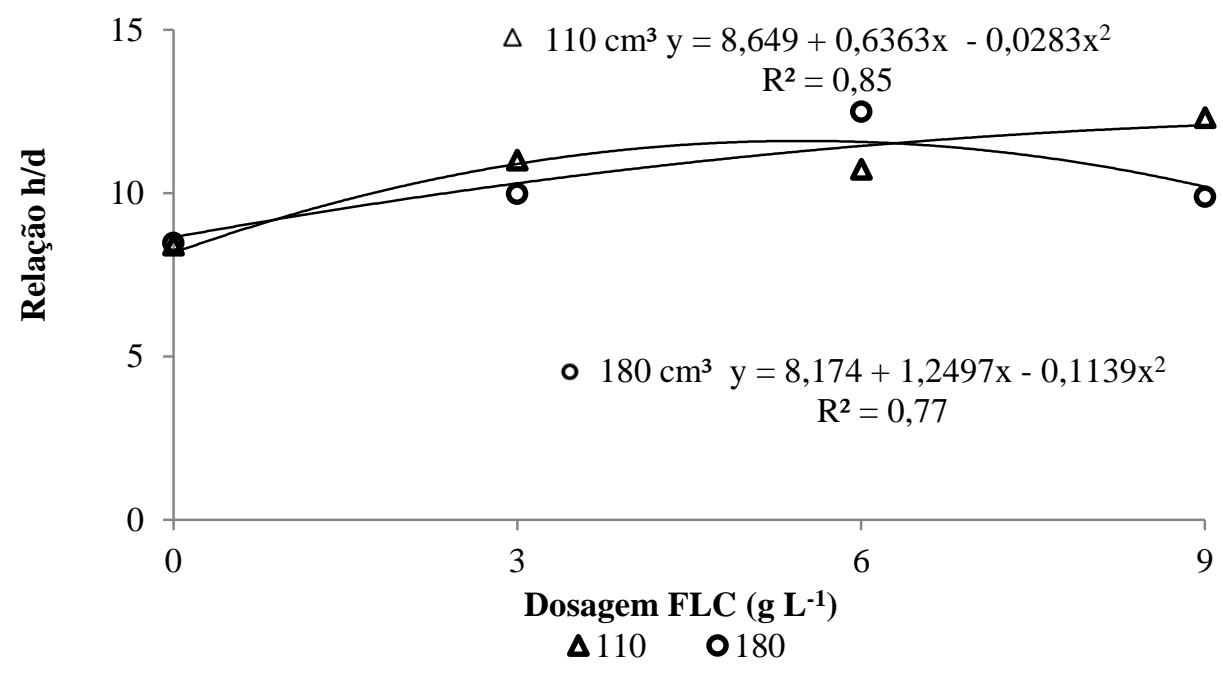

Figura 3: Relação altura/diâmetro do colo de mudas de Mimosa scabrella, em relação à dosagem do fertilizante de liberação controlada (FLC) e volume dos tubetes.

Figure 3: Height/ diameter of Mimosa scabrella plants, in relation to the dosage of the controlled release fertilizer (CRF) and the volume of the tubes. 
A avaliação da altura não pode ser adotada como único critério de qualificação para mudas para o plantio; é necessária, a correlação entre altura e diâmetro de colo, pois podem estar sendo expedidas plantas com o sistema radicular ruim e diâmetros não adequados (que possibilitarão falta de rigidez da haste), e qualidade de parte aérea não desejada.

Entre as mudas cultivadas nesse experimento, as que tiveram melhor comportamento em relação à altura e diâmetro foram aquelas produzidas em tubetes de $180 \mathrm{~cm}^{3}$ com a dosagem de $6 \mathrm{~g} \mathrm{~L}^{-1}$. No entanto, para a maioria das espécies nativas, a metodologia para a produção de mudas em tubetes ainda necessita estudos para a determinação do volume ideal do recipiente bem como a dosagem e métodos de fertilização adequados. $\mathrm{O}$ volume do recipiente está diretamente ligado ao volume para o crescimento radicular, portanto, limitante ao crescimento da planta como um todo (REIS et al., 1989), e indiretamente, afeta a densidade de crescimento, ou seja, a área disponível para cada muda, alterando desta forma, o potencial e o hábito de crescimento da muda (MEXAL e LANDIS, 1990).

\section{Conclusões}

As doses de adubo de liberação lenta influenciaram o crescimento inicial das mudas de bracatinga cultivadas em sistema de tubete contendo substrato comercial. $\mathrm{O}$ maior crescimento das mudas de bracatinga foi obtido com o uso de tubetes de $180 \mathrm{~cm}^{3}$ e $6 \mathrm{~g} \mathrm{~L}^{-1}$ de fertilizante de liberação controlada.

\section{Referências}

BARBOSA, Z:; SOARES, I. CRISÓSTOMO, L. A. Crescimento e absorção de nutrientes por mudas de gravioleira. Revista Brasileira de Fruticultura, Jaboticabal, v.25, n.3. 2003.

BRONDANI, G, E. et al. Fertilização de liberação controlada no crescimento inicial de angico-branco. Scientia Agraria, v.9, n.2, p.167-176, 2008.

CARNEIRO, J. G. A. A influência dos fatores ambientais, das técnicas de produção sobre o desenvolvimento de mudas florestais e a importância dos parâmetros que definem sua qualidade. In: Anais do Simpósio sobre florestas plantadas nos neotrópicos como fonte de energia. Anais... Viçosa, Brasil. p.10-24. 1983.

CARNEIRO, J. G. de A. Produção e controle de qualidade de mudas florestais. Curitiba: UFPR/FUPEF; Campos: UENF, 1995. 451p.

CARNEIRO, R. M.; ALMEIDA Jr, A. R. de.; KAGEYAMA, P. Y.; DIAS, I. de S. Importância da dormência das sementes na regeneração da bracatinga - Mimosa scabrella, Benth. Piracicaba, IPEF, Circular Técnica 149: 1-10. 1982.

CARPANEZZI, O. T. B. Produtividades florestal e agrícola em sistemas de cultivo da bracatinga (Mimosa scabrela Benthaml em Bocaiúva do Sul, região metropolitana. Dissertação (Mestrado). Piracicaba: ESALO, 1994. 77p.

FERMINO, M. H. O uso da análise física na avaliação da qualidade de componentes e substratos. In: FURLANI, A. M.C. et al. Caracterização, manejo e qualidade de substratos para a produção de plantas. Campinas: Instituto Agronômico, p.29-37, 2002.

FERREIRA, D. F. Sisvar: a computer statistical analysis system. Ciência e Agrotecnologia (UFLA), v. 35, n.6, p. 1039-1042, 2011.

GONÇAlVES, J. L. M. et al. Produção de mudas de espécies nativas: substrato, nutrição, sombreamento e fertilização. 2000. In: GOLÇALVES, J. L. M.; BENEDETTI, V. (Orgs.). Nutrição e fertilização florestal. Piracicaba: 2005. p.309-350.

JESUS, R. M. de; MENANDRO, M. de S. Efeito 
do tamanho do recipiente, tipo de substrato e sombreamento na produção de mudas de louro (Cordia trichotoma (Vell.) Arrab.) e gonçaloalves (Astronium fraxinifolium Schott). IPEF, Piracicaba, n. 37, p. 13-19, 1987.

JOSÉ, A. C; DAVIDE, A. C.; OLIVEIRA, S. L. Produção de mudas de aroeira Schinus terebinthifolia Radd para recuperação de áreas degradadas pela mineração de bauxita. Cerne, v.11, n.2, p.187-203, 2005.

KÄMPF, A.N.; FERMINO, M.H. Substratos para plantas. A base da produção vegetal em recipientes. Gênesis, Porto Alegre, Brasil. 312 p. 2000.

KRATZ, D.; WENDLING I. Produção de mudas de Eucalyptus dunnii em substratos renováveis. Floresta v. 43, n.1, p. 125-136. 2013.

LORENZI, H. Árvores brasileiras: Manual de identificação e cultivo de plantas arbóreas nativas do Brasil. Nova Odessa: Plantarum, 1998. 352p.

MARCHIORI, J. N. C. Dendrologia das Angiospermas: leguminosas. 2. ed. Santa Maria: Ed. da UFSM, 2007.

MENDONÇA, V.; RAMOS, J. D.; DANTAS, D. J.; MARTINS, P. P. C.; GONTIJO, T. C. A.; $\mathrm{PIO}, \mathrm{R}$. Efeito de doses de Osmocote e dois tipos de substratos no crescimento de mudas do Mamoeiro Formosa. Ceres, v. 51, n. 296, p. $467-$ 476, 2004.

MEXAL, J.G.; LANDIS, T.D. Target seedling concepts: height and diameter. In: Target seedling symposium, meeting of the western forest nursery associations, general technical report, USDA, 1990, Oregon. Proceedings... Oregon/USDA, 1990. p.17-37.

MORAES NETO, S.P. et al. Fertilização de mudas de espécies arbóreas nativas e exóticas. Revista Árvore, v. 27, n. 2, p. 129-137, 2003.
MOREIRA, F. M. S.; MOREIRA, F. W. Característica de germinação de 64 espécies de leguminosas florestais nativas da Amazônia, em condições de viveiro. Acta Amazônica, Manuas, v. 26, n. 1/2, p. 3-16, 1996.

QUEIROZ, J. A. L.; MELÉM JÚNIOR, N. J. Efeito do tamanho do recipiente sobre o desenvolvimento de mudas de açaí (Euterpe oleracea Mart.). Revista Brasileira de Fruticultura, v.23, n.2, p.460-462, 2001.

REIS, G. G. et al. Crescimento de Eucalyptus camaldulensis, E. grandis e E. cloesiana sob diferentes níveis de restrição radicular. Revista Árvore, v.13, n.1, p.1-18, 1989.

REIS, M.G.F. et al. Crescimento e forma do fuste de mudas de jacarandá-da-bahia (Dalbergia nigra Fr. Allem.), sob diferentes níveis de sombreamento e tempo de cobertura. Revista Árvore, n. 15: n.1, p. 23-24, 1991.

ROSA, F. C. Superação da dormência de sementes e cultivo In vitro de bracatinga (Mimosa scabrella Bentham benth.). 2009. 49

p. Dissertação (Mestrado em Engenharia Florestal). Universidade Federal de Santa Maria, Santa Maria, 2009.

SHARMA, G. C. Controlled-release fertilizers and horticultural applications. Scientia Horticulturae, n. 11, p.107-129, 1979.

SIMÕES, D., SILVA, R.B.G., SILVA, M.R. Composição do substrato sobre o desenvolvimento, qualidade e custo de produção de mudas de Eucalyptus grandis Hill ex. Maiden $\mathrm{x}$ Eucalyptus urophylla s. T. Blake. Ciência Florestal, v. 22, n.1, p. 91-100, 2012.

STURION, J. A.; ANTUNES, B. M. A. Produção de mudas de espécies florestais. In: GALVÃO, A,P.M. Reflorestamento de propriedades rurais para fins de produtivos e ambientais. Colombo: EMBRAPA Florestas, 2000. p. 125-150. 